\title{
A Digital Archive System for Preserving Audio and Visual Space
}

\author{
Makoto Uesaka, Yusuke Ikegaya, and Tomohito Yamamoto \\ College of Information Science and Human Communication, \\ Kanazawa Institute of Technology \\ 7-1 Oogigaoka, Nonoichi, Ishikawa, 921-8501 Japan \\ tyama@neptune.kanazawa-it.ac.jp
}

\begin{abstract}
A digital archive system has been widespread in various fields because it can preserve precious cultural heritage, books, pictures or videos without any deterioration. Moreover, preserving its information on the web, a digital archive system can share a lot of things between general users, and can pass them down new generation easily. In this research, we focus on spatial information of a place or an event which can provide high presence and retrieve personal memories, and develop a digital archive system which can preserve such kind of spatial information.
\end{abstract}

Keywords: Digital Archive, Omnidirectional image, Multi-channel audio, Spatial information.

\section{Introduction}

A digital archive system has been widespread in various fields because it can preserve historical and cultural heritage such as paintings, pictures or sculptures without any deterioration [1]-[4]. Moreover, by providing its data on the web, a digital archive system realizes to share a lot of things between general users, and pass them down next generation. However these digital archives, especially archives provided on the web are likely to be composed of a flat picture and stereo sound. As a result, users can not get as same reality or presence as real things.

In the field of Virtual Reality (VR), to solve this problem, some researches preserve historical heritage by high realistic way and display them by special display system. For example, Abe et. al. have preserved "Maijishan Grotto" in China by stereo camera and displayed them by stereo graphics [5].

In addition to such the research, not only precious historical heritage but also daily life of all around the world has been archived. For example, Watanabe et. al. have archived personal data of people in Tuvalu and reported daily life of Tuvalu where has suffered from sea surface elevation by global warming [6].

In the research of the digital archive of historical heritage, it is possible to preserve and display them in high realistic way. However such the system tends to need expensive equipments for measuring and displaying. Moreover, the high realistic 
display tends to need large space for setting up and does not have mobility. As a result, only precious historical heritage can be archived in the system. On the other hand, in the research of the digital archive of daily life, it provides only photograph, video or text on the web. Therefore it is difficult to feel the reality of peoples' daily life deeply.

In this research, we develop the digital archive system which provides high realistic information without any expensive equipments but mobile devices. In our system, archived data are provided from website, and which can display an omnidirectional image using WebGL and stereo sound using HTML 5. Users also can download the archived contents into our audio-visual display [7], and enjoy them in high realistic way. Moreover, for the archive system, we shoot some contents which are not historical heritage but personally or locally important scenery and space.

\section{A Digital Archive System}

\subsection{System Overview}

Our digital archive system is composed of two components (Fig.1). One is web system which provides archived contents. The other is spatial audio-visual display which realizes to reproduce downloaded contents.

Users can enjoy archived contents by the procedure below;

1. Users access our web site from local PC.

2. Selecting an archive from lists, and users preview it on the browser.

3. Downloading a preferred archive to local PC, and users set up archived data into our display system and enjoy them.

Server side of the web system generates a web page dynamically to client side. Client side realizes to preview and download contents and so on. The spatial audio-visual display is composed of head mount display (HMD) and multiple mobile devices, and which can reproduce downloaded contents by high realistic representation.

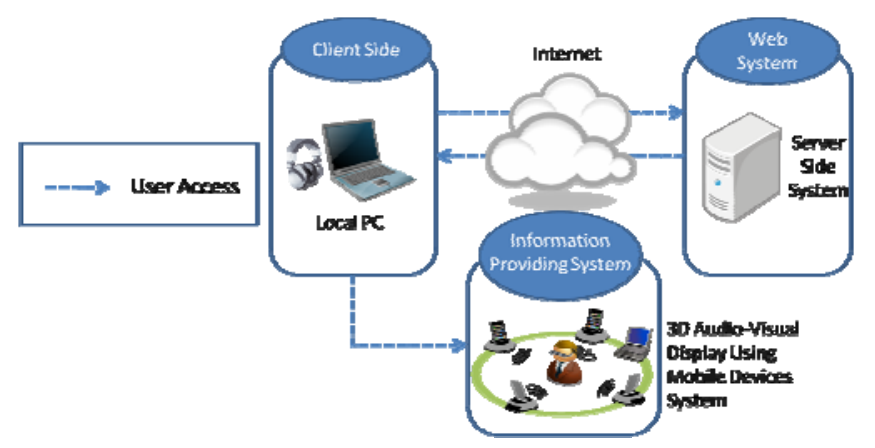

Fig. 1. System Overview 


\subsection{Client and Server Side of the Web System}

Fig.2 shows a top page of a web site. The top page has a function that realizes to search contents from map of Hokuriku area in Japan, or by title, tags and created date. Fig.3(a),(b) show preview images of archived contents. An image of Fig.3(a),(b) derived from a same omnidirectional image but different view angle. In this preview mode, users can see archived space and listen to archived surround sound easily. In this system, archived image is mapped into sphere model, and virtual camera is arranged in center of sphere by using WebGL. If users want to see omnidirectional view, users just click and drag on the displayed image. Moreover, sound data correspond to movement of omnidirectional view (sound data have its own position data in the sphere). If user moves the direction of view, direction and volume of sound also are changed by using API of HTML5.

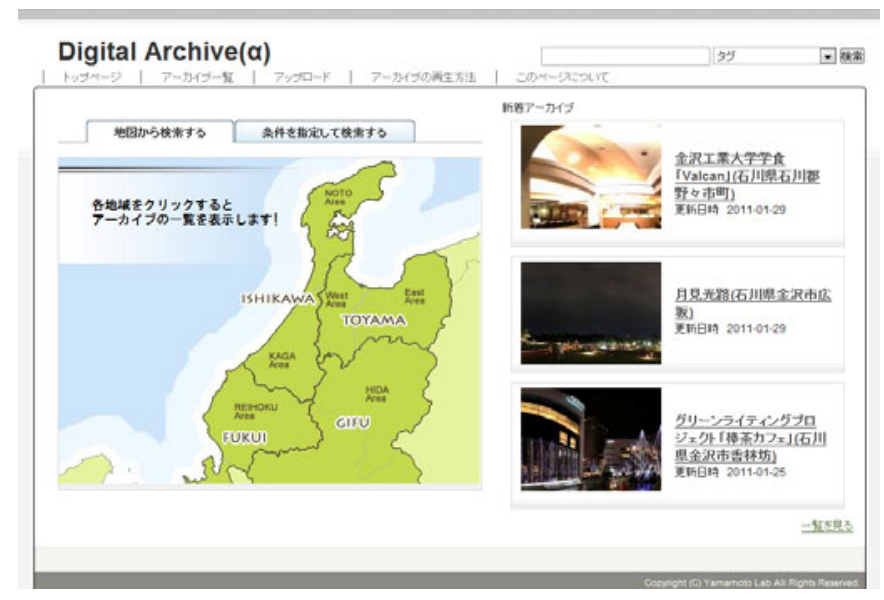

Fig. 2. Top page of web site

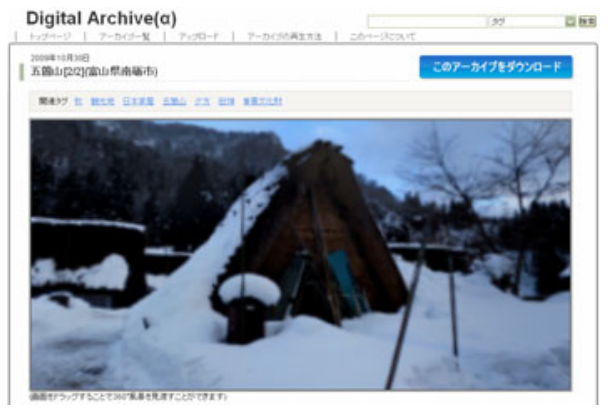

(a)

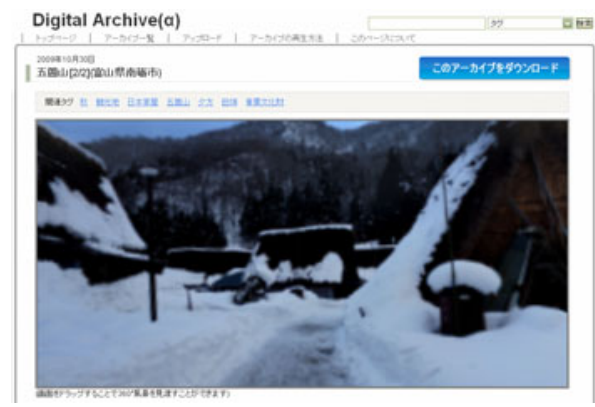

(b)

Fig. 3. Preview page 
Server side of web system is composed of Fedora for the OS, MySQL for the database server, Apache for HTTP server. In server side, generating dynamical page, uploading and downloading process and searching function are implemented using PHP.

\subsection{Spatial Audio-Visual Display System}

To enjoy archived contents in high realistic way, users download contents in local PC, and set up them into our audio-visual display system [7]. Our system is composed of multiple terminals such as a PC or an iPhone as shown in Fig.4. Each terminal is connected to LAN and these are divided into one server, one visual client and multiple sound clients. In this system, at first, the server simulates an archived space. After simulation, the server transmits the information of the virtual space to terminals by UDP multicast. Then, terminals present the high presence to a user using received information.

To reproduce spatial sound, sound clients calculate distance decrement of sound using three positions (a listener's position, a sound source's position and a sound client's position). Using these positions, the system calculates the volume in real time and reproduces a spatial sound by the volume difference of each client.

To represent spatial view, visual client uses a head mount display and a webcamera. For presenting spatial view, the visual client detects user's posture by head tracking sensor, and reflects it to viewing angle in the virtual space.

This system is basically implemented as a software level, and allows any device to become the terminal if the device can be connected to network. Therefore, using devices such as desktop, laptop, or smart phone which have been widespread, our system can be built relatively inexpensive.

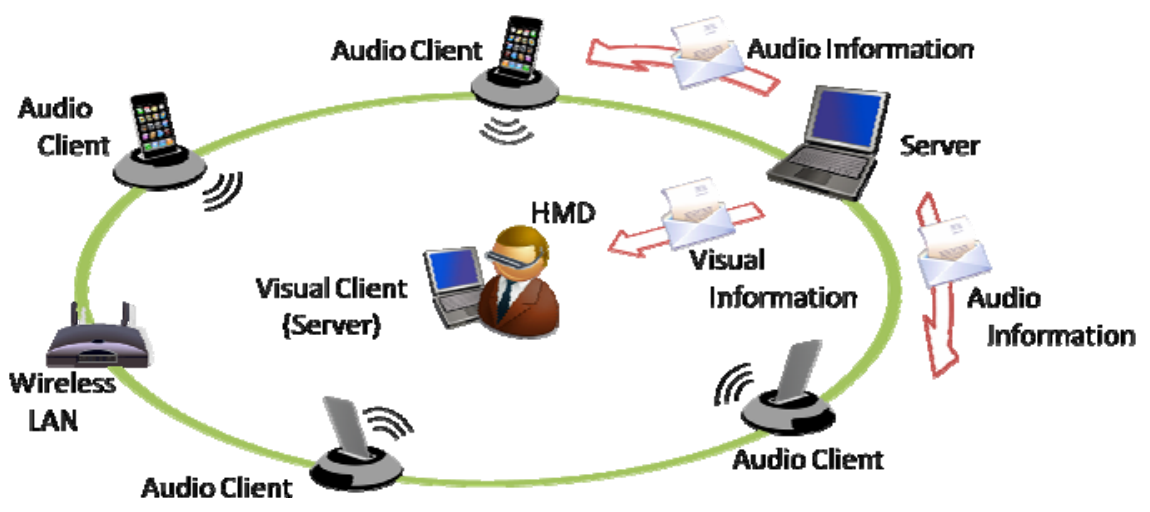

Fig. 4. Spatial audio-visual display 


\section{Contents}

Digital archive systems developed so far have mostly focused on highly valuable heritage such as historical sculptures or fine arts. One reason why such heritage has been archived is that it has been very expensive to create such archives.

However in the world, there are a lot of precious things to archive such as rural beautiful scenery, local festival or events, or personally valuable space. Some of these scenery, events tend to exist in a moment, or tend to fade away if population of its area decrease in the future. In this research, we focus on such things and archive them in low cost way, and provide in highly realistic way.

Concretely, in this research, we have archived beautiful rural scenery of Hakusan area in Ishikawa, and daily student life of Kanazawa Institute of Technology.

In Hakusan area, a population decreases year by year, because there are few jobs in the area. Therefore some villages will be vanished in the future, and beautiful rural scenery called "Satoyama (a very special type of natural environment that cannot exist without moderate intervention of human beings)" also will be vanished. To archive such rural part contributes to preserve the area itself, and if the scenery will be lost, it is possible to tell how this area was to next generation.

Students have a lot of enjoyable, miserable or emotional experience in their student life. Ordinary, they can remember fragments of such memories by photograph or video. If our system provides where they were or how they were in high realistic way, they can experience as if they came back to their student life. Moreover, after student became a parent, they can tell children their student life.

\subsection{Making an Omnidirectional Image and Video}

Fig.5(a) shows all of shooting kit of an omnidirectional image (a general digital camera, a panoramic camera mount and a tripod). To make an omnidirectional image, at first, it is necessary to take pictures by rotating a camera mount by 30 degree. Next, to angle a camera mount up and downward, and take pictures by rotating a mount. Finally, to take a picture of the top. By using these taken pictures (about 40 pictures), an omnidirectional image is created by editing soft (Panoweaver). Fig.6 shows pictures before editing and Fig.7 shows a created omnidirectional image.

To make an omnidirectional video, Ladybug2 (PointGreyResearch) is used. Fig.5(b) shows shooting kit. This 6 lens camera can create omnidirectional video at real time (4.7mega pixel per image).

\subsection{Making Spatial Sound}

In this research, R-09HR (Roland) and a general headphone were used to record sound. However, if it is possible to record sound source clearly in WAVE format, any device is acceptable. Recorded sound sources are placed in virtual sphere in which a created omnidirectional image is mapped. After that, audio data and visual data integrated into one file set (contents information is described in XML format). When reproducing contents, our audio-visual display reads its XML data and simulates archived space. 


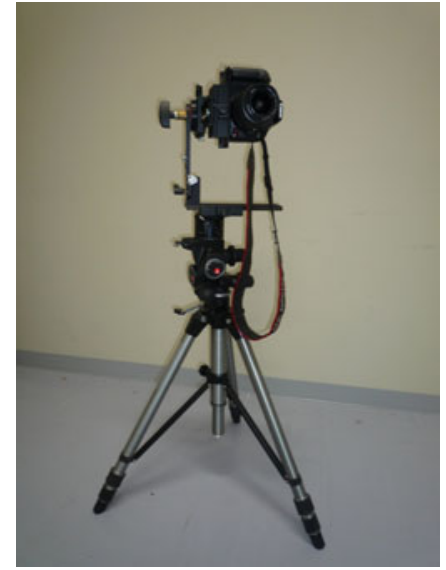

(a)

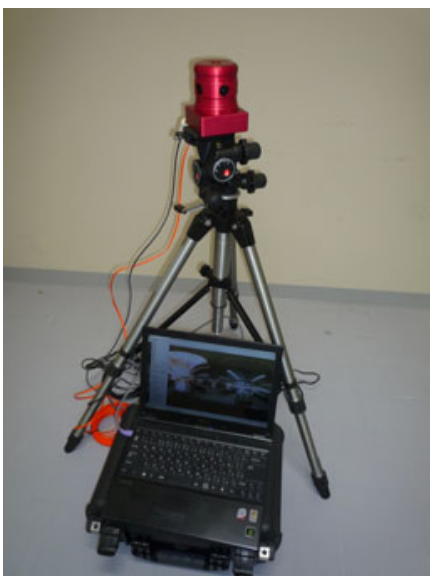

(b)

Fig. 5. Shooting equipments of an omnidirectional image and video

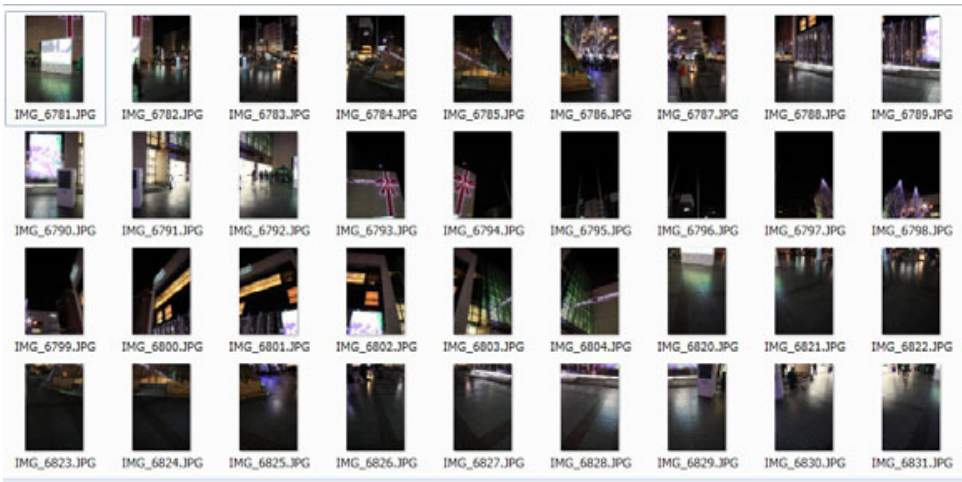

Fig. 6. Unidirectional pictures

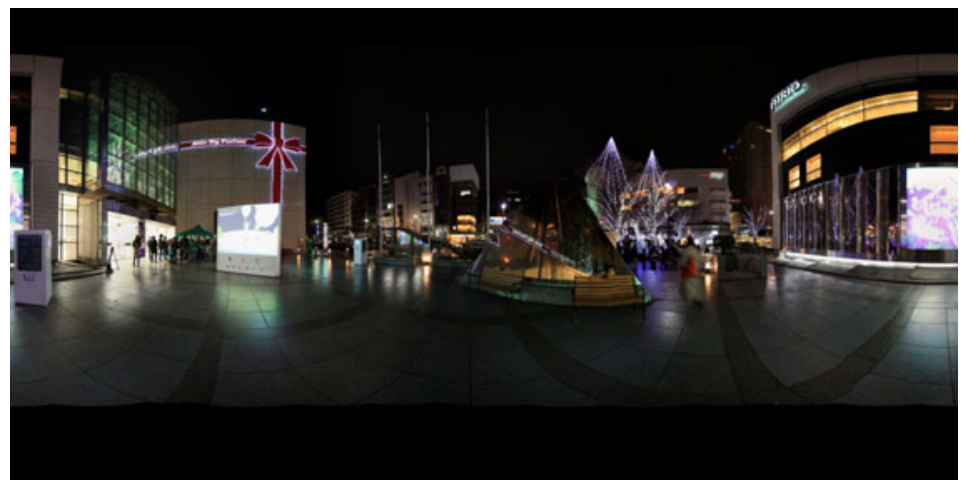

Fig. 7. An omnidirectional Image 


\subsection{Examples of a Digital Archive}

Fig.8-10 show examples of archived contents. Fig.8 shows old Japanese houses called "Gasshou zukuri" which are composed of woods and straw. It is necessary a lot of cost to maintain these houses. However, in this area, young generation go out cities to get jobs, and only elder generation stay. Therefore it is very difficult to keep this scenery in the next generation.

Fig.9 shows Satoyama in Hakusan. Satoyama is very popular Japanese scenery in the old days. In Satoyama, famers cultivated their vegetables and cut firewood for stove in winter. However such famers are rare nowadays and Satoyama vanishes year by year.

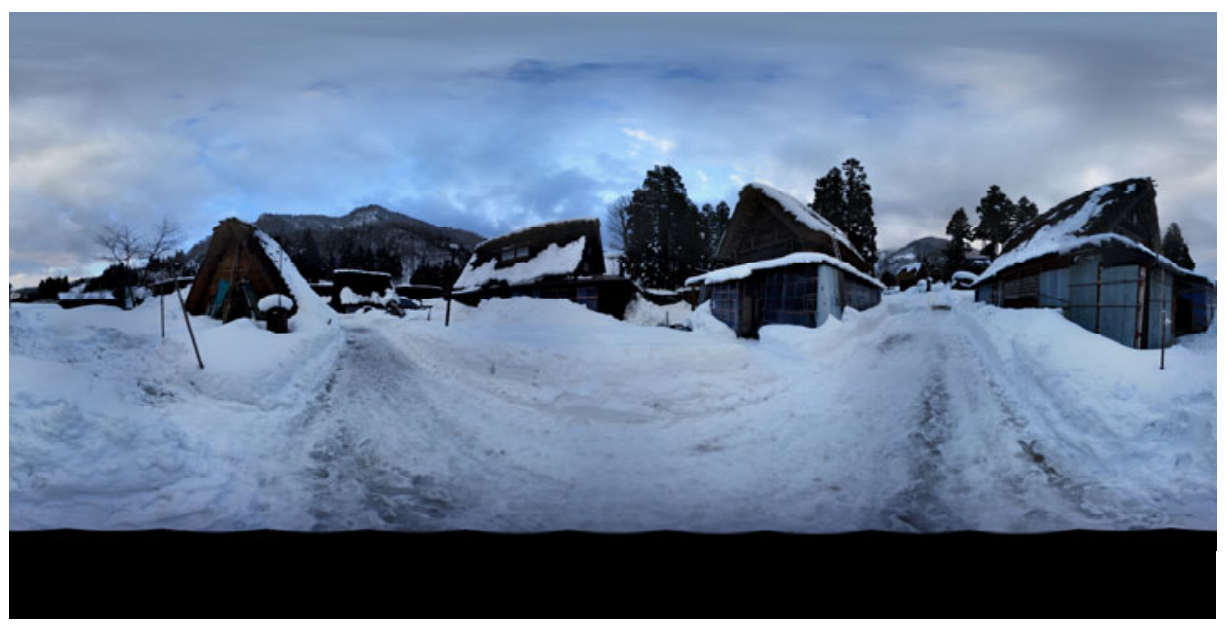

Fig. 8. An archive of "Gasshou zukuri” houses in Gokayama

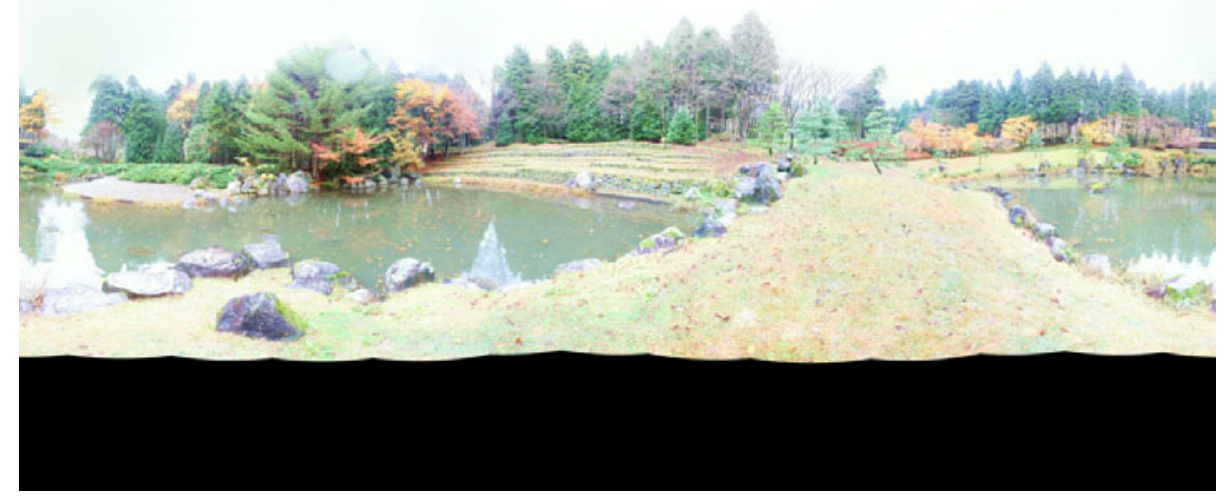

Fig. 9. An archive of "Satoyama" in Hakusan 
Fig.10 shows students' project “Tukimi Kouro" of Kanazawa Institute of Technology. In this project, students light up various places in Kanazawa city by handmade lumps. All of students made a lot of effort in this project. Therefore, this archive would remember their students' life vividly.

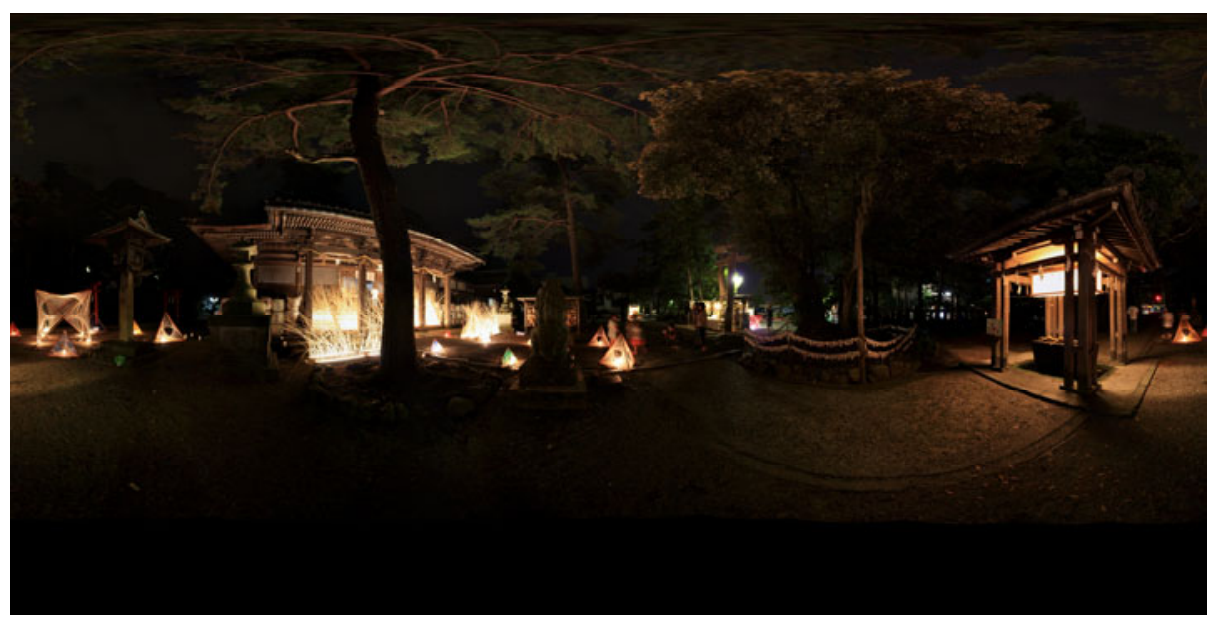

Fig. 10. An archive of "Tsukimi Kouro" at Ishiura Shrine in Kanazawa

\section{Conclusion}

In this research, we developed a digital archive system which realize to preserve high realistic spatial information and to provide it at low cost. Moreover we created some local and personal archived contents. In future works, we will increase contents and release the web sites in public. Moreover we will develop the web system to create an omnidirectional view and surround audio for general users to create high realistic spatial archives.

\section{References}

1. Nagasaki Archive, http://nagasaki mapping.jp/

2. World Digital Library, http: / / www. wdl .org/en/

3. National Archives of Japan, http://www.digital.archives.go.jp/

4. Daijyouji Temple Digital Museum of the Maruyama School, http: / /museum.daijishan.or.jp

5. Abe, N., Kawai, T., Ohya, J., Zha, H., Ando, M.: Digital Archiving of Maijishan and Stereoscopic VR Content. TVRSJ 4(3), 275-282 (2009) (in Japanese)

6. Suzuki, M., Watanabe, Y., Endo, S., Watanabe, H.: Tuvalu Visualization Project. SIGGRAPH ASIA 2009, Sketches, Article No.259 (2009)

7. Takahashi, K., Yamamoto, T.: 3D Audio-Visual Display Using Mobile Devices. In: ACM SIGGRAPH (2010) (Posters) 